\title{
In-Situ Electron Microscopy: a Practical Tutorial
}

\author{
Eric A. Stach
}

School of Materials Engineering, Purdue University, West Lafayette, IN 479907

Advances in imaging and diffraction techniques, sample preparation methods and new holder developments have created substantial recent interest in dynamic electron microscopy. This tutorial will present a general overview of the various categories of in-situ electron microscopy studies, with an emphasis on the classes of materials science problems that can be solved with each approach. It is hoped that participants will walk away with an understanding of how powerful this technique can be, given proper care with respect to experimentation. Considerable emphasis will be placed on ways that the technique can be utilized to determine quantitative information about the physical processes involved, as opposed to merely qualitative descriptions.

A number of in-situ techniques are well established and have been applied to materials problem solving for many decades. These include heating the sample to elevated temperature, cooling it to liquid nitrogen temperature, unixially straining the sample to observe deformation modes and subjecting the sample to changes in environment. More recent developments have extended in-situ studies across nearly all the primary physical science stimuli, as well as offering substantial improvements in experimental approaches towards the more established techniques. Additionally, the ability to combine in-situ studies with other advances in microscopy - e.g. electron holography, atomic-resolution STEM, aberration-corrected microscopy - have lead to remarkable advances in the field. Examples of the utilization of each of these types of in-situ experimentation will be presented, relying heavily on published results from leaders in the field.

As a technique, in-situ TEM studies can suffer from unwanted artifacts introduced by the thin foil geometry. I will discuss various sample preparation and experimental strategies that can be used to minimize (but not entirely mitigate) these concerns. In many cases, it is possible to utilize microfabrication methods common in the semiconductor electronics industry to create samples of controlled size and shape. This can lead to situations where thin foil effects are no longer a concern, have been appropriately minimized, or are at least well understood. Additionally, the increased access to focused ion beam based sample preparation techniques has had a dramatic impact, allowing direct exploration of the effects of sample thickness on the resulting sample response.

Additionally, dramatic increases in computation power and improvements in off-the-shelf software allow rapid and fully automated data extraction from videotape captures of experiments. I will demonstrate how these methods can be utilized to extract quantitative data from in-situ experimentation.

Finally, it is worth noting that the development of a full array of in-situ methods is well beyond the resources of most laboratories. This fact is well recognized, and as a result, nearly all of these techniques are available at one or more of the Department of Energy's Electron Microbeam Characterization Centers. I will outline which resources are available at which location, as well as the procedures to gain access to each. 